\section{UCDNN}

LIBRARY
University of Connecticut OpenCommons@UConn

$3-2012$

\title{
Electrophysiological Responses to Threat in Youth with and without Posttraumatic Stress Disorder
}

Damion J. Grasso

University of Connecticut School of Medicine and Dentistry

Follow this and additional works at: https://opencommons.uconn.edu/uchcres_articles

Part of the Medicine and Health Sciences Commons

\section{Recommended Citation}

Grasso, Damion J., "Electrophysiological Responses to Threat in Youth with and without Posttraumatic Stress Disorder" (2012). UCHC Articles - Research. 157.

https://opencommons.uconn.edu/uchcres_articles/157 


\title{
Electrophysiological Responses to Threat in Youth with and without Posttraumatic Stress Disorder
}

\author{
Damion J. Grasso ${ }^{a}$ and Robert F. Simons ${ }^{b}$ \\ aUniversity of Connecticut Health Center, Department of Psychiatry, 263 Farmington Avenue, \\ Farmington, CT 06030. Telephone: (860) 301-7641. FAX: (860) 679-4326. dgrasso@uchc.edu \\ bUniversity of Delaware, Department of Psychology, 108 Wolf Hall, Newark, DE 19716. \\ Telephone: (302) 831-2389. FAX: (302) 831-1041. rsimons@psych.udel.edu
}

\section{Abstract}

The current study was designed to examine event-related brain potentials and autonomic responses to pictures indicating threat, relative to non-threat, and acoustic startle reflexes in traumatized youth diagnosed with PTSD, relative to non-exposed children, before and after receiving psychotherapy. Children in the control group were individually yoked and demographically matched to the PTSD group. Both groups displayed enhanced late positive potentials and more prolonged heart rate deceleration to pictures indicating threat, relative to non-threat, and larger skin conductance responses to pictures indicating threat, relative to non-threat, at time one. At time two, controls appeared to habituate, as reflected by an overall attenuated skin conductance response, whereas the PTSD group showed little change. Across time points the PTSD group exhibited greater acoustic startle reflexes than the control group. Psychotherapy and symptom reduction was not associated with electrophysiology. Drawing from the adult literature, this study was an attempt to address the scarcity of research examining electrophysiological irregularities in childhood PTSD. The overall results suggest that children and adolescents allocate more attention to threat-related stimuli regardless of PTSD status, and exaggerated startle and a possible failure to habituate skin conductance responses to threat-related stimuli in youth with versus without PTSD.

\section{Keywords}

Posttraumatic Stress Disorder; Electrophysiology; Trauma-Exposure; Autonomic; Event-Related Brain Potential; Acoustic Startle

\begin{abstract}
Marked physiological reactions to trauma reminders, hypervigilance, and exaggerated startle responses constitute a substantial portion of symptom criteria in the diagnosis of Posttraumatic Stress Disorder (PTSD). In fact, among the 17 symptoms that currently characterize PTSD, 35\% are associated with physiological reactivity, officially recognized as a defining feature of the disorder in the DSM-III-R (APA, 1987). Physiological reactivity, however, is largely oversimplified in the DSM, where it is presented as a unitary construct when in fact it reflects structurally and functionally distinct operations of the central nervous
\end{abstract}

(c) 2012 Elsevier B.V. All rights reserved.

Correspondence to: Damion J. Grasso.

Publisher's Disclaimer: This is a PDF file of an unedited manuscript that has been accepted for publication. As a service to our customers we are providing this early version of the manuscript. The manuscript will undergo copyediting, typesetting, and review of the resulting proof before it is published in its final citable form. Please note that during the production process errors may be discovered which could affect the content, and all legal disclaimers that apply to the journal pertain.

The authors do not have any financial disclosures to report. 
system (CNS) and the sympathetic (SNS) and parasympathetic (PNS) divisions of the autonomic nervous system (ANS). These systems are intertwined in a complex and dynamic interrelationship in which antagonistic influences co-operate to maintain homeostasis in the face of environmental demands ${ }^{1}$. Dysregulation among these systems can result in failure to adaptively respond to these demands, a condition that may be inherently tied to psychopathology, and for the purpose of this study, PTSD. ${ }^{2,3}$ Studying these systems in the context of perceived threat in individuals with and without PTSD may help to better understand the array of physiological characteristics associated with the disorder.

The fear response begins at the level of the CNS. Within a couple of hundred milliseconds the CNS recognizes an environmental stressor as a potential threat and triggers ANS responses. Because of the speed at which this occurs, the measurement of event-related brain potentials (ERPs), known for their fine temporal resolution, is a fruitful method of examining information processing. A recent meta-analytic review of ERP studies involving PTSD highlighted a number of irregularities in sensory information processing in adult participants. ${ }^{4}$ Two of the reviewed studies linked PTSD with an enhanced threat-elicited P300, an ERP positivity observed approximately $300 \mathrm{~ms}$ post-stimulus onset at midline parietal sites and associated with attention allocation. 5,6 The classic P300 is characterized by a positive deflection that occurs approximately 300 milliseconds post-stimulus onset. The range within which the $\mathrm{P} 300$ can be found is dependent on experimental parameters, sometimes resulting in a short range (e.g., 300-400 ms) and other times a more pervasive positive wave extending beyond $400 \mathrm{~ms}$. In fact, the P300 is considered part of a larger family of positive slow waves observed at later latencies (> $400 \mathrm{~ms}$ ) and dependent on the experimental task. Late positive deflections that sometimes extend from 400-1000 ms or more are generally referred to as late positive potentials (LPPs). In such cases, the P300 is no longer considered a separate entity, but rather a constituent of the LPP. From here onward we will refer to late positive slow waves more broadly as LPPs.

LPPs are thought to reflect attention allocation and motivational significance, ${ }^{7}$ and this is evident in the classic oddball task. The oddball task is a paradigm in which participants are instructed to attend to target or oddball stimuli within a series of non-target or irrelevant stimuli, and to indicate whether each stimulus is a target or not by pushing one of two buttons. Larger LPP amplitudes to target stimuli have been associated with superior attention and memory performance. ${ }^{4}$ The modified oddball task is one in which participants attend to target (oddball) stimuli within a series of irrelevant stimuli or distractors, including threat-related stimuli, and indicate via button press whether each stimulus is a target or nontarget. In non-traumatized adults, target stimuli elicit larger LPPs than distractors; however, adults with military-related PTSD exhibit LPPs to threat-related distractors that are comparable to those elicited by targets. ${ }^{4-6}$ No studies have examined the LPP in relation to childhood PTSD and in the context of threat; however, studies comparing maltreated and non-maltreated children have reported larger LPPs to angry faces, relative to neutral and positive faces, in maltreated children, suggesting a tendency to allocate more attention to social threat. 8,9

There is also a sizeable literature on SNS and PNS correlates of PTSD in adult populations. A meta-analysis and a number of recent studies document heightened autonomic reactivity and exaggerated startle in traumatized adult patients diagnosed with PTSD. ${ }^{10-18}$ These include greater skin conductance responses (SCRs) and heart rate responses (HRR) to standardized and idiographic trauma cues, as well as slower skin conductance habituation slopes and exaggerated startle responses to startle probes. ${ }^{10}$ A separate line of work focuses on HR deceleration in response to approaching threat, which invokes increased vigilance as the individual prepares for mobilization and survival motivated action. ${ }^{19}$ In a number of studies, individuals with PTSD, compared to controls, have been shown to exhibit greater 
HR deceleration in response to approaching threat and greater subsequent HR acceleration when threat is perceived as imminent. ${ }^{11,20,21}$

Despite the sizeable literature on autonomic irregularities in adults with PTSD, comparable research on children and adolescents lags behind. The few studies pertaining to childhood PTSD report mixed results. A study of preschool children who had survived a motor vehicle accident reported elevated heart rate in response to a 3-min conversation about a hypothetical car crash, relative to controls, with no significant difference between those with and without PTSD. ${ }^{22}$ A prospective study found that elevated heart rate immediately following a traumatic injury predicted PTSD 6-months later; ${ }^{14}$ however another study by this group did not replicate this finding. ${ }^{23}$ In contrast to studies of adults, one study found no evidence of enhanced acoustic startle in adolescent girls with full or partial PTSD, relative to non-traumatized controls. ${ }^{24}$ Another study reported diminished acoustic startle reflexes in a small sample of children who developed PTSD after witnessing a sniper attack compared to non-exposed children. ${ }^{25}$ Clearly, efforts towards reconciling these variable findings and establishing a stronger knowledge base of physiological reactivity in youth populations are needed.

An additional question is whether there are meaningful changes in physiological responses to threat in individuals after completion of a behavioral treatment that is effective in reducing self-reported symptoms of PTSD. Addressing this question may be important in understanding the relationship between physiological responses and subjective symptoms of distress, as well as beginning to pursue whether specific patterns of physiological responses to threat in individuals with PTSD precede or follow development of the disorder. For example, if physiological responses to threat are associated with PTSD and there are significant changes in these responses as a function of treatment completion and success, this may suggest value in pursuing prospective examinations of one or more of these correlates as potential endophenotypes or sequelae of trauma-related symptomatology. Clinical implications may include supplementing self- or other-reported assessment of symptoms with an objective and relatively non-intrusive biological measure, which, depending on whether it is a premorbid risk-factor or a consequence of the disorder, might also be used to predict or measure treatment effectiveness, respectively. Using psychophysiological methodology to test changes in fear reactivity before and after behavioral intervention is not a novel approach; however, is sparse in the literature. In one of the earlier studies, Lang, Melamed, and $\mathrm{Hart}^{26}$ reported reduced autonomic activity (i.e., reduced HRRs, greater habituation) after systematic desensitization in a sample of college students with snake phobia, with greater reductions predicting better treatment outcome. Two, more recent, studies have examined autonomic reactivity in adults with PTSD before and after receiving exposure-based treatment. One of these studies found that reduced SCRs to trauma-related stimuli from pre- to post-treatment was associated with greater symptom reduction in combat veterans. ${ }^{27}$ Another study of combat veterans reported that SCRs, HRRs, and frontalis electromyogram (EMG) responses to script-driven imagery of traumatic events decreased from pre- to post-treatment, but only for scripts relating to events targeted for desensitization. ${ }^{28}$ No known studies have examined differences in ERPs following treatment for PTSD, nor have there been studies examining the relation between treatment outcome and electrophysiology in traumatized youth with PTSD.

In summary, there has been substantial effort to identify physiological irregularities in adult PTSD populations, and especially in male combat veterans. This work highlights irregularities that include enhanced autonomic sensitivity, startle reflexes, and ERP indices of attention allocation to perceived threat in individuals with PTSD compared to those without PTSD. The same research in child and adolescent PTSD populations is scarce, and that which does exist provides mixed results. In addition, to our knowledge, only two studies 
have examined whether autonomic irregularities in adults with combat-related PTSD change over the course of treatment, both reporting significant change relative to a control group. There are no known studies examining startle reflexes or threat-elicited ERPs before and after treatment in adults or children. A better understanding of the relationship between PTSD symptoms and physiology, and the malleability of observed physiological irregularities following effective treatment, may have implications for how PTSD is conceptualized, assessed, and ultimately addressed in treatment. This was our motivation for undertaking the current study.

The current study was designed to examine LPPs, SCRs, and HRRs to threat-related pictures, relative to non-threat pictures, presented in the context of a modified oddball task, and acoustic startle reflexes in trauma-exposed children and adolescents with PTSD, relative to non-exposed children, before and after receiving exposure-based treatment. We rigorously controlled for individual differences between groups by; (a) individually yoking the non-exposed and PTSD groups so that for each child in the PTSD group there was a child in the non-exposed group that was assessed before and after a period of time that approximated the length of treatment for the child in the PTSD group, and (b) demographically matching the pairs on age, gender, and self-identified race. PTSD symptoms were assessed by self-report before and after treatment to determine whether treatment resulted in significant symptom reduction. In addition, parent-reported internalizing and externalizing behavior problems, associated with PTSD symptoms, demonstrated significant symptom reduction in the PTSD group, and not the non-exposed group. We hypothesized that traumatized youth with PTSD, compared to non-exposed youth would exhibit larger amplitude LPPs, HRRs, and SCRs to threat-related and target pictures relative to neutral and positive pictures, and larger amplitude startle reflexes to acoustic startle probes. We also hypothesized that traumatized youth with PTSD would show reductions in LPPs, HRRs, and SCRs to threat-related pictures and reductions in acoustic startle reflexes over the course of therapy, relative to the non-exposed control group.

\section{Methods and Materials}

\section{Participants}

Participants were 19 trauma-exposed children (ages 10-17) receiving Trauma-Focused Cognitive Behavioral Therapy (TF-CBT) to treat PTSD, and 19 non-exposed control children, who were individually yoked and demographically matched to the trauma-exposed children on age, gender, self-identified race, and length-of-time between experimental sessions (pre- and post-treatment of the PTSD group). Participants were $63.2 \%$ female. Selfidentified race included 55.3\% European American, 31.6\% African American, 7.9\% Hispanic American, and 5.2\% Biracial. Age ranged from 9.6 to 16.27 years with a mean of $13.48 \pm 2.2$ in the PTSD group and $13.49 \pm 2.02$ in the non-exposed group. Annual household income was significantly larger in the control group (mean $\$ 56,263.16 \pm$ $\$ 28,195.43$ ) than the PTSD group (mean $\$ 35,652.63 \pm \$ 27,107.84$ ), $t(36)=-2.3, p=.028$. Percent of caregivers currently employed was $73.68 \%$ in the PTSD group and $68.42 \%$ in the non-exposed group. None of the control families reported receiving welfare, whereas $26.32 \%$ in the PTSD group were current recipients. All of participating caregivers in the non-exposed group had a high school diploma or above, and the majority $(78.95 \%)$ of caregivers in the PTSD group did as well. Percent of participating caregivers married or living with a partner was $42.11 \%$ in the PTSD group and $73.68 \%$ in the non-exposed group.

\section{Diagnostic Classification}

PTSD was assessed by a trained Master's level clinician (supervised by a licensed child psychologist) using a semi-structured diagnostic interview, the Schedule for Affective 
Disorders and Schizophrenia for School-Age Children, Present and Lifetime version (KSADS-PL), ${ }^{29}$ a semi-structured diagnostic interview that includes diagnosis-specific impairment ratings, generates DSM-IV diagnoses, and divides symptoms into a screening interview and five diagnostic supplements. Twenty percent of the interviews conducted in the larger effectiveness study were double-coded yielding good inter-rater agreement (kappa $=.93$ ).

For seven children, PTSD was secondary to sexual abuse, followed by physical abuse ( $n=$ $5)$, witnessing domestic violence $(n=5)$, witnessing community violence $(n=1)$, and hearing about the violent death or serious injury of a loved one $(n=1)$. The mean length of time between trauma exposure and baseline assessment was $2.56 \pm 3.4$ years. All children in the PTSD group endorsed exposure to multiple potentially traumatic events (mean $4.05 \pm$ 1.9). Fourteen children in the PTSD group presented with comorbid psychiatric disorders at baseline. These included: Major Depressive Disorder (MDD; $n=6$ ), Depressive Disorder NOS (DDNOS; $n=1)$, Overanxious Disorder $(n=2)$, Attention Deficit/Hyperactivity Disorder (ADHD; $n=3$ ), and Oppositional Defiant Disorder (ODD; $n=3$ ). In addition, nine children met partial criteria for MDD $(n=1)$, DDNOS $(n=2)$, Bipolar Disorder NOS $(n=$ 2), Separation Anxiety Disorder $(n=2)$, Simple Phobia $(n=1)$, Obsessive Compulsive Disorder $(n=1)$, ADHD $(n=4)$, Conduct Disorder $(n=3)$, and ODD $(n=3)$. Eleven children endorsed a history of suicidal ideation, four with suicidal gestures, and one with a suicide attempt. One child was receiving psychopharmacological treatment for MDD and ADHD, which consisted of bupropion and atomoxetine, respectively.

Four control participants presented with a full DSM-IV Axis I diagnosis including Generalized Anxiety Disorder $(n=1)$ and $\operatorname{ADHD}(n=3)$. Two control participants met criteria for a partial diagnosis including Bipolar Disorder NOS $(n=1)$ and Separation Anxiety Disorder $(n=1)$. No suicidal behavior was reported. Finally, two control participants were prescribed stimulant medication to treat ADHD (i.e., methylphenidate, lisexamfetamine dimesylate).

\section{Symptom Severity}

PTSD symptom severity was assessed using the UCLA PTSD Reaction Index for DSM-IV (UCLA PTSD RI). ${ }^{30}$ The UCLA PTSD RI has excellent psychometric properties with good convergent validity ( 0.70 with the K-SADS-PL) and a sensitivity of 0.93 and specificity of 0.87 in diagnosing PTSD. ${ }^{31}$ The test-retest reliability coefficient was reported to be 0.84 in one study. ${ }^{31}$ The Child Behavior Checklist $6-18(\mathrm{CBCL})^{32}$ was used to measure parentreported internalizing and externalizing behavior problems in both groups. Items on the CBCL are scored on a 3-point Likert scale ranging from 0 ("Not True") to 2 ("Very True") of the child. Scores were used to establish two broad-band scales: Internalizing (withdrawn, anxious/depressed, somatic complaints) and Externalizing (delinquent, aggressive) behavior problems. The CBCL has been used with acceptable levels of reliability (range $=.84-.98$ ), content, and criterion validity to measure behavior problems of children ages $6-18$ years from diverse racial and ethnic backgrounds. ${ }^{32}$

\section{Procedure}

Recruitment-Trauma-exposed participants were recruited from a larger communitybased effectiveness study of TF-CBT in Delaware, funded by the Substance Abuse and Mental Health Services Association (SAMHSA). Inclusion criteria for the PTSD group was dependent on those used in the larger effectiveness study, except for a narrower age range and more stringent diagnostic criteria, and included the following: (a) age was between 10 and 17 years at pre-treatment; (b) child and parent were English speaking; (c) child was insured by Medicaid or had no insurance; (d) exposure to a potentially traumatic event 
reported by the child or legal guardian, or confirmed by police report or child protective services, if child maltreatment or victimization was involved; (e) met criteria for a full DSM-IV diagnosis of PTSD, and (f) availability of a participating caregiver who was not the perpetrator of the traumatic event. Exclusion criteria were the following: (a) prior DSM-IV diagnosis of mental retardation or significantly impaired cognitive functioning; (b) active psychosis; (c) current alcohol or substance abuse; and (d) acute risk of suicide or homicide.

Control participants were recruited from the New Castle County Delaware area via flyers and advertisements posted locally in the newspaper and in public places. Neighborhoods and communities from which PTSD participants resided were targeted for recruitment of control participants in order to help facilitate demographic matching. Inclusion criteria were the following: (a) age was between 10 and 17 years at baseline; (b) child and parent were English speaking; and (c) availability of a caregiver participant. Exclusion criteria were the same employed for the PTSD group except for child exposure to a potentially traumatic event per child- or parent-report. Prospective control participants meeting inclusion criteria and their legal guardians were initially screened using the child and parent versions of the UCLA PTSD RI to rule-out trauma-exposure. If no exposure was reported, the K-SADS-PL was administered to examine additional exclusion criteria (i.e., active psychosis, current alcohol or substance abuse, significant cognitive impairment). Prospective control participants matched with PTSD participants on age in years ( \pm 6 months), gender, and selfidentified race were retained for the current study, while non-matching participants were invited to partake in a related study.

All recruitment procedures and experimental methods were approved by the University of Delaware Human Subjects Review Board.

Trauma-Focused CBT-TF-CBT is a 12- to 16-session intervention designed to reduce child traumatic stress. TF-CBT educates the child and caregiver about trauma and PTSD, and teaches stress-reduction, emotion regulation, and cognitive coping skills. The core of TF-CBT involves creation of a trauma narrative, which functions as a means of therapeutic exposure and facilitates emotional and cognitive processing of the event. The efficacy of TF-CBT has been demonstrated several randomized controlled trials. 3334

Laboratory Task and Stimuli-PTSD and control participants engaged in an oddball task, a paradigm that has been used in numerous ERP experiments and is a well-documented method for eliciting the late positive potential (LPP) component. ${ }^{35}$ Participants were instructed to view a series of pictures and to press the number one key on the number pad of a standard keyboard, labeled 'Yes', if the picture was a target or the number two key on the number pad, labeled ' $N o$ ', if the picture was not a target. Target pictures (30)1 consisted of animals from the IAPS and occurred $25 \%$ of the time. Non-target pictures (90) consisted of positive (30)2, neutral (30)3, and threat-related pictures (30)4 chosen from the IAPS. ${ }^{36}$ Threat-related pictures were those rated high on arousal and negative valence and illustrated content conveying threat, such as physical assault, weaponry, and threatening faces. No animal pictures were included in the threat-related set. Pictures illustrating mutilations and erotica were eliminated from the stimulus set. Positive and threat-related pictures were

\footnotetext{
1 p1602, p1920, p1604, p1910, p1670, p1590, p1463, p1812, p1721, p1510, p1450, p1710, p1601, p1460, p1560, p1750, p1810, p1740, p1603, p1540, p1500, p1660, p1440, p1650, p1720, p1610, p1620, p1900, p1600, p1640

2p8260, p5831, p5700, p5600, p5891, p5760, p8032, p8040, p5628, p2070, p8162, p8117, p7502, p5390, p5470, p1999, p5410, p8021, p5300, p7325, p8370, p8496, p8210, p5623, p5890, p8080, p7330, p8031, p7270, p5480

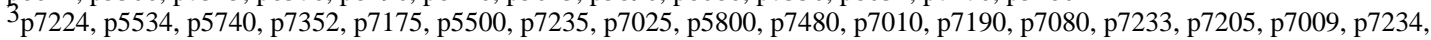
p7004, p7560, p7207, p7284, p7035, p7150, p7140, p7030, p7320, p7006, p7217, p7570, p5750

${ }_{4}^{4} 6210, p 9041, p 6243, p 6250, p 3500, p 6200, p 6230, p 6260, p 6560, p 6244, p 2100, p 6510, p 6242, p 6212, p 6190, p 6211, p 6530$, p6300, p6830, p2682, p6360, p6313, p6314, p2130, p6350, p6370, p2681, p6570, p2120, p2691
} 
matched by arousal ratings. Each picture type occurred $25 \%$ of the time. The inclusion of threat-related pictures was a modification of the oddball paradigm and has been used by a number of researchers interested in examining the LPP in the context of PTSD. Pictures were presented for $200 \mathrm{~ms}$ with a constant inter-stimulus interval of $5000 \mathrm{~ms}$ using Presentation software (Neurobehavioral Systems, Inc.). Electrophysiological measures (SCRs, HRRs, and ERPs) were time-locked to the pictures.

During inter-stimulus intervals, participants were instructed to fixate on the ' $x$ ' in the middle of the computer screen and told that they would occasionally hear loud sounds (i.e., startle probes) through a pair of earphones, and to do their best to ignore the sounds. Startle probes were 50-millisecond, 100-decibel bursts of white noise with instantaneous rise time created with Audacity Inc. software (www.audacity.sourceforge.net) and presented over Phillips SBC HN110 earphones with external amplification. There were a total of 10 startle probes delivered during randomly selected inter-stimulus intervals that succeeded neutral pictures. Startle probes randomly occurred at 1000, 2000, 3000, or $4000 \mathrm{~ms}$ after the start of each of the 10 inter-trial intervals. Startle probes and recording of startle reflexes did not overlap with recordings of other physiological measures. Using 10 inter-trial intervals to deliver startle probes capitalized on study design and minimized the length of the laboratory session, which may have been prohibitive for some of the participating youth and families if startle reflexes were measured in a separate task.

\section{Electrophysiological Recording and Data Reduction}

Electroencephalogram (EEG) - All data were recorded by the principal investigator and trained research assistants. Two tin $9 \mathrm{~mm}$ cup disk electrodes (ECI) were attached on the left and right mastoids (M1 and M2, respectively). Two $\mathrm{Ag} / \mathrm{AgCl}$ miniature electrodes (MedAssociates) were attached $1 \mathrm{~cm}$ above and below subjects' left eye to record the vertical electrooculogram (EOG). A clip electrode functioning as a ground was attached to subjects' left ear. EEG recordings were taken from frontal $(\mathrm{Fz})$, central $(\mathrm{Cz})$, and parietal $(\mathrm{Pz})$ areas along the midline using an ECI electrocap. All electrode impedances were below $10 \mathrm{k} \Omega$ and the data from all channels were recorded using a Grass Model 7P1 Low Level DC Preamplifier and Model 7D Driver Amplifier (bandpass $=.1-35 \mathrm{~Hz}$ ). During the recording, EEG activity from $\mathrm{Fz}$ and $\mathrm{Pz}$ reference sites were referenced to $\mathrm{Cz}$. All bioelectric signals were digitized on a laboratory microcomputer using VPM software. ${ }^{37}$ The EEG was sampled at $200 \mathrm{~Hz}$. Data collection began $500 \mathrm{~ms}$ prior to picture presentation and continued $1000 \mathrm{~ms}$ after picture onset.

Offline, EEG data reduction was performed using EEGLAB 6.01 (http://www.sccn.ucsd.edu/eeglab) ${ }^{38}$ running under Matlab 7.6 (The Mathworks). All data were rereferenced from $\mathrm{Cz}$ to the average-mastoid reference. All trials were inspected visually, and only trials without artifact were retained. The EEG data for each trial was corrected for vertical EOG artifact by employing Independent Component Analysis (ICA). ICA decomposed each trial's data into five components. Components representing EOG artifact were identified and rejected by calculating Pearson-Product Moment correlations between each component and the EOG channel, then determining the highest correlation. These determinations were also corroborated by visual inspection. ERPs were based on a minimum of 20 trials. Finally, using EEGLAB 6.01, artifact-free EEG data were regenerated by back-projecting the remaining ICA components, which was accomplished by multiplying the selected component activities with the reduced component-mixing matrix. Single trial EEG data were lowpass filtered at $20 \mathrm{~Hz}$ with a 51-weight FIR digital filter. Stimulus-locked ERPs were averaged separately for each stimulus type. A baseline equal to the average activity in a $200 \mathrm{~ms}$ window prior to picture onset was subtracted from each data point after 
picture onset. Late positive potentials were defined as the average amplitude within a window of $500-1000 \mathrm{~ms}$ following stimulus onset.

Skin Conductance-Skin conductance responses were recorded using VPM software at a sampling rate of $50 \mathrm{~Hz}$. Med Associates Standard $\left(0.5 \mathrm{~cm}^{2}\right) \mathrm{Ag} / \mathrm{AgCl}$ electrodes were placed on the thenar and hypothenar eminence of the palm of the non-dominant hand with TD-246 Skin Conductance Electrode Paste from Discount Disposables used as an electrolyte. The skin conductance signal was transduced by a Coulbourn Model S21-22 constant voltage (0.5 V) skin conductance coupler. Data was graphically displayed on a trial-by-trial basis so as to visually identify response onset and the largest peak occurring within $0.5-4 \mathrm{~s}$ post stimulus onset. SCRs were defined as the difference, in $\mu$ Siemens, between the peak and onset points.

Electrocardiogram (ECG) - The raw ECG signal was recorded using VPM software from electrodes placed approximately 6-inches below the left armpit (i.e., midaxial line on the lateral aspect of the chest) and on the chest approximately 6-inches from the collarbone (i.e., lower peristernal region). ECG signals were amplified using a Grass Model 7P1 Low Level DC Preamplifier and Model 7D Driver Amplifier (bandpass $=.1-35 \mathrm{~Hz}$ ) and then processed by a series of Coulbourn logic modules employing threshold detection and shaping prior to the online computation of inter-beat intervals to the nearest $1 \mathrm{~ms}$. Inter-beat intervals were converted to heart rate in beats per minute per real time epoch $(500 \mathrm{~ms})$. When epochs contained portions of two beats, each rate was weighted according to the fraction of the epoch that it occupied. ${ }^{39}$ Heart-rate waveforms were generated by deviating half-second averages during a $5000 \mathrm{~ms}$ epoch from the half-second average immediately preceding stimulus onset.

Electromyogram (EMG)—Raw EMG signals from the right ocularis oculi region (bandpass $=8-250 \mathrm{~Hz}$ ) were fed into a Coulbourn Contour Following Integrator Model S76-01 (time constant $=10 \mathrm{~ms}$ ) and recorded using VPM software. The EMG channel was digitized at $1000 \mathrm{~Hz}, 50 \mathrm{~ms}$ prior and $250 \mathrm{~ms}$ after startle probe onset. Eyeblink reflex amplitudes were measured base-to-peak using a Fortran IV program ${ }^{40}$ that identifies the integrated EMG response to the startle probe and rejects invalid data (e.g., excessive baseline shift, peak latency exceeds peak window criterion, onset to peak time too long, out of range). Plotted waveforms were visually examined to ensure program validity.

\section{Statistical Analysis}

Independent groups t-tests, chi-square goodness-of-fit tests, and repeated-measures ANOVAs were conducted using IBM SPSS Statistics (Version 19). Skewness and kurtosis were examined for all dependent variables to determine if data were normally distributed. In the event that normality could not be assumed (i.e., skewness $>2$; kurtosis $>2$ ), data were transformed (i.e., in order of square root, $\log 10$, and inverse transformations) and reexamined. Partial eta squared values were reported to demonstrate the size of effects such that 0.02 represents a small effect, 0.15 a medium effect, and 0.35 a large effect. ${ }^{41}$ Hochberg's ${ }^{42}$ modified Step-Up Bonferroni procedure was implemented to test pairwise comparisons within each analysis.

\section{Results}

\section{Symptom Severity}

All data from the UCLA PTSD RI and CBCL were normally distributed (skewness < 2; kurtosis <2). The PTSD group had a UCLA PTSD RI mean of $34.21 \pm 3.58$, which was significantly reduced at post-treatment (mean 17.16 \pm 13.83$), t(14)=5.02, p<.001$. The PTSD group (mean $55.79 \pm 13.75$ ) had significantly greater Internalizing behavior problem 
$\mathrm{t}$-scores than the control group (mean 45.21 \pm 12.03 ), $t(36)=2.74, p=.010, d=.82$. Additionally, the PTSD group (mean $54.94 \pm 13.75)$ had significantly greater externalizing behavior problem t-scores than the control group (mean 45.58 \pm 11.31 ), $t(36)=2.94, p=$. $028, d=.74$. The PTSD group showed a significant reduction in internalizing behavior problem t-scores at time two (mean 54.94 \pm 13.75 ), $t(14)=4.46, p<.001$, as well as externalizing behavior problem t-scores (mean 55.79 \pm 13.75 ) $, t(14)=3.76, p=.001$; however, there were no significant changes in internalizing or externalizing scores for the control group.

\section{Late-Positive Potential}

Raw waveforms are presented in Figure 1. All data were normally distributed (skewness $<2$; kurtosis $<2$ ). A time point $\times$ site $\times$ picture type repeated-measures ANOVA with mean LPP as the dependent variable revealed a main effect for site, $F(2,56)=52.19, p<.001, \eta_{\mathrm{p}}{ }^{2}=$. $65, \alpha=.99$, a main effect for picture type, $F(3,84)=7.28, p<.001, \eta_{\mathrm{p}}{ }^{2}=.21, \alpha=.98$, and a site $\times$ picture type interaction, $F(6,168)=10.61, p=.005, \eta_{\mathrm{p}}{ }^{2}=.10, \alpha=.92$. Pairwise comparisons indicated that mean LPPs significantly differed among all sites $(p s<.001)$, with the largest mean LPP at Pz, followed by $\mathrm{Cz}$ and Fz. Regarding the picture type effect, LPPs to target and threat-related pictures were significantly greater than LPPs to Positive and Neutral pictures $(p s<.05)$. A simple effects analysis of the site $\times$ picture type interaction indicated that significant differences among picture types occurred at $\mathrm{Pz}(p<.05$; see Figure 2), but not significantly so at $\mathrm{Fz}$ or $\mathrm{Cz}(p s>.1)$.

\section{Heart-Rate Responses}

Raw HRR waveforms are presented in Figure 3. Visual examination of the raw HRR waveforms revealed a quadratic pattern, with HR deceleration observed within a 250-1750 ms time window, followed by a recovery period observed within a 2250-3250 ms time window. Due to the quadratic nature of the data, analyses were conducted in a piecewise fashion (i.e., deceleration, recovery), as done in previous research. ${ }^{43} \mathrm{HR}$ deceleration was defined as the average change in bpm within a $250-1750 \mathrm{~ms}$ time window, and recovery as the average change in bpm within a 2250-3250 ms time window. All data were normally distributed (skewness $<2$; kurtosis $<2$ ).

A full-factorial repeated measures ANOVA with group type as the between-subject variable, time point and picture type as within-subject variables, and mean HRR in the deceleration period as the dependent variable revealed a main effect for time point, $F(1,24)=8.91, p=$. $006, \eta_{\mathrm{p}}{ }^{2}=.27, \alpha=.82$, such that deceleration was greatest at time two. All other effects were non-significant, $p s>.1$. A full-factorial repeated measures ANOVA with the same independent variables and using mean $\mathrm{HR}$ acceleration in the recovery period as the dependent variable revealed a significant main effect for picture type, $F(3,72)=2.77, p=$. $045, \eta_{\mathrm{p}}{ }^{2}=.08, \alpha=.65$, such that threat-related pictures resulted in less HR acceleration (i.e., less recovery) than other pictures ( $p s<.05)$, and a main effect for group type, $F(1,24)=$ $7.99, p=.009, \eta_{\mathrm{p}}{ }^{2}=.25, \alpha=.77$, such that HR acceleration was greater (i.e., greater recovery) in the PTSD group compared to the control group. Non-significant effects were found for time point and all possible interactions, $p s>.1$.

\section{Skin Conductance Responses}

Raw SCR data are presented in Figure 4. SCR data were positively skewed (skew > 2) and leptokurtic (kurtosis > 2) and so data were normalized using $\log 10$ transformations. A fullfactorial repeated measures ANOVA with group type as the between-subject variable, time point and picture type as the within subject variables, and $\log 10$ transformed SCRs as the dependent variable yielded a main effect for time point $F(1,24)=5.03, p=.034, \eta_{\mathrm{p}}{ }^{2}=17, \alpha$ $=.58$, a main effect for picture type, $F(3,72)=9.92, p<.001, \eta_{\mathrm{p}}^{2}=.29, \alpha=.99$, and a group 
type $\times$ time point $\times$ picture type interaction, $F(3,72)=5.95, p=.001, \eta_{\mathrm{p}}{ }^{2}=.2, \alpha=.95$. The main effect for group type and all other possible interactions were non-significant, $p s>.1$. The main effect for time point indicated significantly smaller SCRs at time two compared to time one $(p<.001)$. The main effect for picture type indicated that SCRs were significantly larger to threat-related pictures compared to all other pictures $(p s<.01)$. The group type $\times$ time point $x$ picture type interaction indicated that while the control group exhibited greater increases between threat-related pictures and other picture types at time one, there were no significant differences among picture types at time two; however, the PTSD group continued to show increased SCRs to threat-related pictures, relative to other picture types, at time two (see Figure 5).

\section{Acoustic Startle Reflexes}

Acoustic startle reflex data, in microvolts, were leptokurtic (kurtosis > 2) and were normalized with square-root transformation. There was a significant main effect for group type indicating greater startle reflexes for the PTSD group (mean $607.61 \mu \mathrm{v} \pm 637.97 \mu \mathrm{v}$ ) relative to the control group (mean $289.05 \mu \mathrm{v} \pm 378.32 \mu \mathrm{v}$ ), $F(1,30)=4.51, p=.042, \eta_{\mathrm{p}}{ }^{2}=$. $13, \alpha=.54$. Non-significant effects were found for time point and the time point $\times$ group type interaction.

\section{Discussion}

This study was the first to rigorously examine electrophysiological autonomic and central nervous system responses to threat-related stimuli in children and adolescents with PTSD compared to non-traumatized, yoked, and demographically matched controls, before and after exposure-based treatment of the PTSD g roup. Both groups displayed greater LPPs to threat-related and target pictures, relative to positive and neutral pictures, during the modified oddball task, with no significant difference between time one and time two. This suggests that children and adolescents, regardless of trauma-exposure and PTSD, show greater attention allocation to threat, even when utilizing attentional resources to search for task-related target stimuli. While this finding is contrary to our hypothesis that the PTSD group would exhibit relatively greater LPPs to threat compared to the non-exposed group, it is consistent with a large body of work demonstrating a normative tendency to allocate more attention to highly arousing and affective stimuli relative to stimuli perceived as less arousing/affective. ${ }^{36}$ Our finding no ERP differences between the PTSD and non-exposed groups, however, is in contrast to two studies reporting enhanced LPPs to threat-related and target stimuli, relative to irrelevant stimuli, in military veterans with PTSD compared to veterans without PTSD. ${ }^{5,6}$ To note, the relationship between early processing of threat stimuli in military-related samples and PTSD may not generalize to children and adolescents, who have not undergone intense combat training to respond to imminent danger in the context of a routine level of threat and who are at very different developmental stages.

Our hypotheses that the PTSD group would exhibit greater initial HR deceleration and greater subsequent acceleration, relative to the non-exposed group, were not supported. In both groups, HR acceleration was better conceptualized as a recovery period, where HR returned to baseline. Neither group showed the marked HR acceleration that would be expected to follow HR deceleration should threat have been perceived as more proximal or imminent. One possibility is that omitting IAPS pictures that were not developmentally appropriate for this age range (e.g., mutilations, blood) resulted in a selection of threatrelated pictures that were not potent enough to elicit this level of threat. Another possibility is that the time window chosen to observe HR was not long enough to reveal acceleration above baseline. Ultimately, we opted to interpret this stage as a recovery period. As such, viewing threat-related pictures was associated with a delayed recovery from initial deceleration, relative to other pictures, suggesting that threat-related pictures invoke a more 
sustained period of attention. This is compatible with research showing more pronounced HR deceleration in normative samples of children when processing unpleasant or frightening pictures compared to pleasant or neutral pictures. ${ }^{44}$

SCRs to threat-related pictures were larger than responses to other pictures at time one in both groups; however, this difference was more robust in the control group. At time two, the control group appeared to completely habituate to the threat-related pictures, and no longer exhibited larger SCRs to threat. In contrast, the PTSD group maintained this effect, exhibiting relatively larger SCRs to threat-related pictures at time two. In effect, while the control group appeared to habituate to the threat-related pictures on their second engagement with the task, the PTSD group did not habituate, which is consistent with the adult literature suggesting a deficient ability in individuals with PTSD to demonstrate expected habituation to repeated stimuli. ${ }^{10}$ While we were not specifically assessing habituation rates, nor included hypotheses pertaining to habituation, this interpretation seems more reasonable than the alternative; that involvement in trauma-focused treatment somehow compromised habituation to threat stimuli. In either case, this may be an important avenue for future research.

Children with PTSD showed greater acoustic startle reflexes than control children, albeit the effect was small. This is in line with the adult literature showing elevated acoustic startle reflexes in patients with anxiety disorders, including PTSD. ${ }^{10,45}$ Our findings disagree with one study that failed to find enhanced startle reflexes in adolescent girls with PTSD symptoms,${ }^{24}$ and another study that reported diminished startle reflexes in a small sample of children with PTSD related to a sniper attack. ${ }^{25}$ The acoustic startle reflex is modulated entirely by the CNS via a relatively simple pathway that receives direct input from the amygdala, a structure that is heavily implicated in fear learning. ${ }^{46}$ Given that the exaggerated startle reflex constitutes its own symptom in the DSM-IV diagnosis of PTSD, it will be especially important to understand the heterogeneity of results reported in the child literature.

Finally, despite that the PTSD group showed significant reductions in self-report PTSD symptoms, as well as parent-reported symptoms of internalizing and externalizing behavior problems, while the non-exposed did not, TF-CBT did not appear to have a noticeable impact on electrophysiology. Given that ERP, HRR, and SCR patterns were comparable between groups at baseline, differences in the PTSD group before and after treatment, however, would not have been readily interpretable. The lack of significant change in acoustic startle reflexes before and after treatment may suggest that exaggerated startle is a premorbid characteristic of PTSD, or more likely reflects the small overall effect of the group difference and insufficient power to detect the even smaller interaction effect.

This study was an initial attempt to address the scarcity of research on electrophysiological correlates of PTSD in children and adolescents, using the adult literature as a theoretical and methodological springboard. The study has limitations. First, the small sample size restricted statistical power and prevented a thorough examination of variables that would be worthwhile to explore in the future (e.g., developmental differences, comorbidity, trauma types). Regarding the non-significant results of the current study, however, power analyses indicated that in order to achieve adequate power to detect main and interaction effects of those magnitudes would necessitate sample sizes ranging from 70 to 2,200, suggesting that our non-significant effects were particularly small; however, we cannot ignore the increased risk of Type II error. Second, a more comprehensive study would require two control groups: a non-traumatized group and a trauma-exposed without PTSD group. As it stands, it cannot be differentiated whether group differences in SCRs and startle reflexes were the result of trauma-exposure, or more specifically, a PTSD diagnosis. Third, in examining 
potential treatment effects, an ideal design would not only control for the passage of time, as the current study had, but also control for any placebo effect. Fourth, the age range of children and adolescents was quite large, from 10- to 17-years-old. There may be important developmental differences that we were not equipped to examine and that may have confounded our results. It will be critical to examine potential age and developmental differences in future studies of this nature. Finally, although standardized threat-related pictures were used, pictures depicting possible inter-partner or community violence may have been more salient to the children exposed to these types of violence. Due to the limited sample size of the current study, differences in physiological responses to threat according to its relation to the target trauma was not possible and is recommended for future work in this area. A particular strength of the current study was the yoked and demographically matched control design, in which each participant receiving treatment was matched to a control participant by gender, race, and approximate age (i.e., \pm 6 -months) and the length of time between pre- and post-treatment for each participant in the control group was approximately equivalent to that of his or her match in the PTSD group (i.e., \pm 2 -weeks). This design minimized random error and potential confounding variables. As practical advice for increasing the likelihood of detecting potential group differences in future studies, we recommend: (a) recruiting a larger sample size, which would increase power and enable an examination of other potential covariates (e.g., gender, age), (b) increasing external validity by utilizing idiographic threat cues (e.g., pictures that relate to the child's target trauma), (c) enhancing the arousing or threatening nature of the experimental context and/or stimuli (i.e., intensifying the experimental manipulation), (d) employing a variety of experimental tasks to identify and take into account possible task-specific effects, and (e) measuring a broader array of physiological indicators so as to establish a fuller picture of nervous system functioning.

Our PTSD sample represented children with significant trauma exposure and serious emotional and behavioral problems. More than half of the sample endorsed three or more exposures to potential trauma. At pre-treatment, the majority of the sample reported suicidal ideation at some point in the past year; with almost half of these children having made a suicidal gesture, and one child having actually attempting suicide. More than a third of the sample met criteria for a comorbid mood disorder. Approximately a third of the sample was living below the poverty line and receiving state and/or federal assistance. These children and families represent a portion of the population with tremendous life challenges and significant clinical and non-clinical needs. Continuing efforts to understand risk factors and sequelae of trauma-related symptomatology in this population, and improve our ability to identify and treat trauma-related problems is of utmost importance.

In conclusion, the current study contributes to our knowledge of electrophysiological correlates of PTSD in children and adolescents, and along with the few existing studies, calls into question the generalizability of physiological irregularities observed in adults with PTSD to children with the disorder. More work will be necessary to reconcile the findings from studies examining electrophysiology in children and adolescents with PTSD and to determine the malleability of physiology in the course of treatment.

\section{Highlights}

- As in adults, children and adolescents exhibited greater late positive potentials and larger skin conductance responses to pictures indicating threat, relative to non-threat.

- Children and adolescents exhibited less recovery from initial heart rate deceleration in response to threat-related pictures, relative to non-threat pictures. 
- At follow-up, skin conductance responses in non-traumatized children and adolescents habituated, whereas children with PTSD maintained larger responses to threat-related pictures compared to non-threat pictures.

- Children with PTSD generated greater acoustic startle reflexes than nontraumatized children.

- Psychotherapy did not appear to influence electrophysiological measures.

\section{Acknowledgments}

This research was funded in part by the National Institute of Mental Health (NIMH; F31 MH 86227-01).

The authors wish to thank the state of Delaware for affording us the time and necessary resources for the completion of this work, as well as the children and families who have generously donated their time.

\section{References}

1. Kandel, ER.; Schwartz, JH.; Jessell, TM. Principles of Neural Science. 4th ed. New York: McGrawHill; 2000.

2. El-Sheikh M, Kouros CD, Erath S, et al. Marital conflict and children's externalizing behavior: Interactions between parasympathetic and sympathetic nervous system activity: Abstract. Monographs of the Society for Research in Child Development. 2009 Apr.74(1):vii. [PubMed: 19302676]

3. McEwen BS. The neurobiology of stress: From serendipity to clinical relevance. Brain Research. 2000 Dec; 886(1-2):172-189. [PubMed: 11119695]

4. Karl A, Malta LS, Maercker A. Meta-analytic review of event-related potential studies in posttraumatic stress disorder. Biological Psychology. 2006 Feb; 71(2):123-147. [PubMed: 15961210]

5. Kimble MO, Fleming K, Bandy C, Zambetti A. Attention to novel and target stimuli in trauma survivors. Psychiatry Research. In Press, Corrected Proof.

6. Attias J, Bleich A, Firman V, Zinger Y. Event-related potentials in post-traumatic stress disorder of combat origin. Biological Psychiatry. 1996 Sep; 40(5):373-381. [PubMed: 8874838]

7. Hajcak G, Dennis TA. Brain potentials during affective picture processing in children. Biological Psychology. 2009; 80(3):333-338. [PubMed: 19103249]

8. Shackman JE, Shackman AJ, Pollak SD. Physical abuse amplifies attention to threat and increases anxiety in children. Emotion. 2007 Nov; 7(4):838-852. [PubMed: 18039053]

9. Pollak SD, Cicchetti D, Klorman R, Brumaghim JT. Cognitive brain event-related potentials and emotion processing in maltreated children. Child Development. 1997 Oct; 68(5):773-787.

10. Pole N. The psychophysiology of posttraumatic stress disorder: A meta-analysis. Psychological Bulletin. 2007 Sep; 133(5):725-746. [PubMed: 17723027]

11. Adenauer H, Catani C, Keil J, Aichinger H, Neuner F. Is freezing an adaptive reaction to threat? Evidence from heart rate reactivity to emotional pictures in victims of war and torture. Psychophysiology. 2010; 47(2):315-322. [PubMed: 20030756]

12. Arditi-Babchuk H, Feldman R, Gilboa-Schechtman E. Parasympathetic reactivity to recalled traumatic and pleasant events in trauma-exposed individuals. Journal of Traumatic Stress. 2009; 22(3):254-257. [PubMed: 19444883]

13. Felmingham KL, Rennie C, Manor B, Bryant RA. Eye Tracking and Physiological Reactivity to Threatening Stimuli in Posttraumatic Stress Disorder. Journal of Anxiety Disorders. 2011

14. Bryant RA, Salmon K, Sinclair E, Psychol M, Davidson P. Heart rate as a predictor of posttraumatic stress disorder in children. General Hospital Psychiatry. 2007 Jan-Feb; 29(1):66-68. [PubMed: 17189749]

15. Guthrie RM, Bryant B. Auditory Startle Response in Firefighters Before and After Trauma Exposure. American Journal of Psychiatry. 2005 Feb; 162(2):283-290. [PubMed: 15677592] 
16. Pineles SL, Mostoufi SM, Ready CB, Street AE, Griffin MG, Resick PA. Trauma Reactivity, Avoidant Coping, and PTSD Symptoms: A Moderating Relationship? Journal of Abnormal Psychology. 2011; 120(1):240-246. [PubMed: 21319932]

17. Griffin MG. A prospective assessment of auditory startle alterations in rape and physical assault survivors. Journal of Traumatic Stress. 2008; 21(1):91-99. [PubMed: 18302176]

18. Pole N, Neylan TC, Otte C, et al. Associations between childhood trauma and emotion-modulated psychophysiological responses to startling sounds: A study of police cadets. Journal of Abnormal Psychology. 2007 May; 116(2):352-361. [PubMed: 17516767]

19. Lang PJ, Davis M, Ohman A. Fear and anxiety: Animal models and human cognitive psychophysiology. Journal of Affective Disorders. Special Issue: Arousal in anxiety. 2000; 61(3): 137-159.

20. Cuthbert BN, Lang PJ, Strauss C, Drobes D, Patrick CJ, Bradley MM. The psychophysiology of anxiety disorder: Fear memory imagery. Psychophysiology. 2003 May; 40(3):407-422. [PubMed: 12946114]

21. McTeague LM, Lang PJ, Laplante MC, Cuthbert BN, Shumen JR, Bradley MM. Aversive imagery in posttraumatic stress disorder: trauma recurrence, comorbidity, and physiological reactivity. Biological Psychiatry. 2010 Feb 15; 67(4):346-356. [PubMed: 19875104]

22. Scheeringa MS, Zeanah CH, Myers L, Putnam F. Heart period and variability findings in preschool children with posttraumatic stress symptoms. Biological Psychiatry. 2004 Apr; 55(7):685-691. [PubMed: 15065300]

23. Nixon RDV, Nehmy TJ, Ellis AA, Ball S-A, Menne A, McKinnon AC. Predictors of posttraumatic stress in children following injury: The influence of appraisals, heart rate, and morphine use. Behaviour Research and Therapy. 2010 Aug; 48(8):810-815. [PubMed: 20537316]

24. Lipschitz DS, Mayes LM, Rasmusson AM, et al. Baseline and Modulated Acoustic Startle Responses in Adolescent Girls With Posttraumatic Stress Disorder. Journal of the American Academy of Child \& Adolescent Psychiatry. 2005 Aug; 44(8):807-814. [PubMed: 16034283]

25. Ornitz EM, Pynoos RS. Startle modulation in children with posttraumatic stress disorder. American Journal of Psychiatry. 1989 Jul; 146(7):866-870. [PubMed: 2742011]

26. Lang PJ, Melamed BG, Hart J. A psychophysiological analysis of fear modification using an automated desensitization procedure. Journal of Abnormal Psychology. 1970; 76:220-234. [PubMed: 5483369]

27. Boudewyns PA, Hyer L. Physiological response to combat memories and preliminary treatment outcome in Vietnam veteran PTSD patients treated with direct therapeutic exposure. Behavior Therapy. 1990 Win;21(1):63-87.

28. Shalev AY, Orr SP, Pitman RK. Psychophysiologic response during script-driven imagery as an outcome measure in posttraumatic stress disorder. Journal of Clinical Psychiatry. 1992 Sep; 53(9): 324-326. [PubMed: 1355475]

29. Kaufman J, Birmaher B, Brent D, et al. Schedule for Affective Disorders and Schizophrenia for School-Age Children-Present and LIfetime Version (K-SADS-PL): initial reliability and validity data. Journal of the American Academy of Child \& Adolescent Psychiatry. 1997; 36(7):980-988. [PubMed: 9204677]

30. Pynoos, R.; Rodriguez, N.; Steinberg, AM.; Stuber, ML.; Frederick, C. The UCLA PTSD Reaction Index for DSM IV. Los Angeles: UCLA Trauma Psychiatry Program; 1998.

31. Steinberg AM, Brymer MJ, Decker K, Pynoos RS. The University of California at Los Angeles Post-Traumatic Stress Disorder Reaction Index. Current Psychiatry Reports. 2004; 6:96-100. [PubMed: 15038911]

32. Achenbach, TM. The Child Behavior Checklist. Burlington: ASEBA, University of Vermont; 2001.

33. Cohen JA, Deblinger E, Mannarino AP, Steer RA. A multisite, randomized controlled trial for children with sexual abuse-related PTSD symptoms. Journal of the American Academy of Child \& Adolescent Psychiatry. 2004 Apr; 43(4):393-402. [PubMed: 15187799]

34. Cohen J, Mannarino AP. Disseminating and implementing trauma-focused CBT in community settings. Trauma, Violence, \& Abuse. 2008; 9(4):214-226. 
35. Luck, SC. An introduction to the event-related potential technique. Cambridge, MA: The MIT Press; 2005.

36. Lang, PJ.; Bradley, M.; Cuthbert, BC. International affective picture system (IAPS): Technical manual and affective ratings. Gainsville: University of Florida, Center for Research in Psychophysiology; 1999.

37. Cook, EWI. VPM Reference Manual. Birmingham: Alabama; 1999.

38. Delorme A, Makeig S. EEGLAB: an open source toolbox for analysis of single-trial EEG dynamics including independent component analysis. Journal of Neuroscience Methods. 2004; 134:9-21. [PubMed: 15102499]

39. Graham FK. Constraints on measuring heart rate and period sequentially through real and cardiac time. Psychophysiology. 1978 Sep; 15(5):492-495. [PubMed: 693763]

40. Balaban MT, Losito B, Simons RF, Graham FK. Off- line latency and amplitude scoring of the human reflex eyeblink with Fortran IV. Psychophysiology. 1986; 23:612.

41. Cohen J. Statistical power analysis for the behavioral sciences. 1988

42. Hochberg Y. A sharper Bonferroni procedure for multiple tests of significance. Biometrika. 1988; 75:800-802.

43. Hodes RL, Cook EW, Lang PJ. Individual differences in autonomic response: Conditioned association or conditioned fear? Psychophysiology. 1985; 22(5):545-560. [PubMed: 4048355]

44. McManis MH, Bradley MM, Berg WK, Cuthbert BN, Lang PJ. Emotional reactions in children: verbal, physiological, and behavioral responses to affective pictures. Psychophysiology. 2001; 38:222-231. [PubMed: 11347868]

45. Grillon C. Startle reactivity and anxiety disorders : Aversive conditioning, context, and neurobiology. Biological Psychiatry. 2002 Nov; 52(10):958-975. [PubMed: 12437937]

46. Garfinkel SN, Liberzon I. Neurobiology of PTSD: A review of neuroimaging findings. Psychiatric Annals. 2009 Jun; 39(6):370-372. 376-381. 

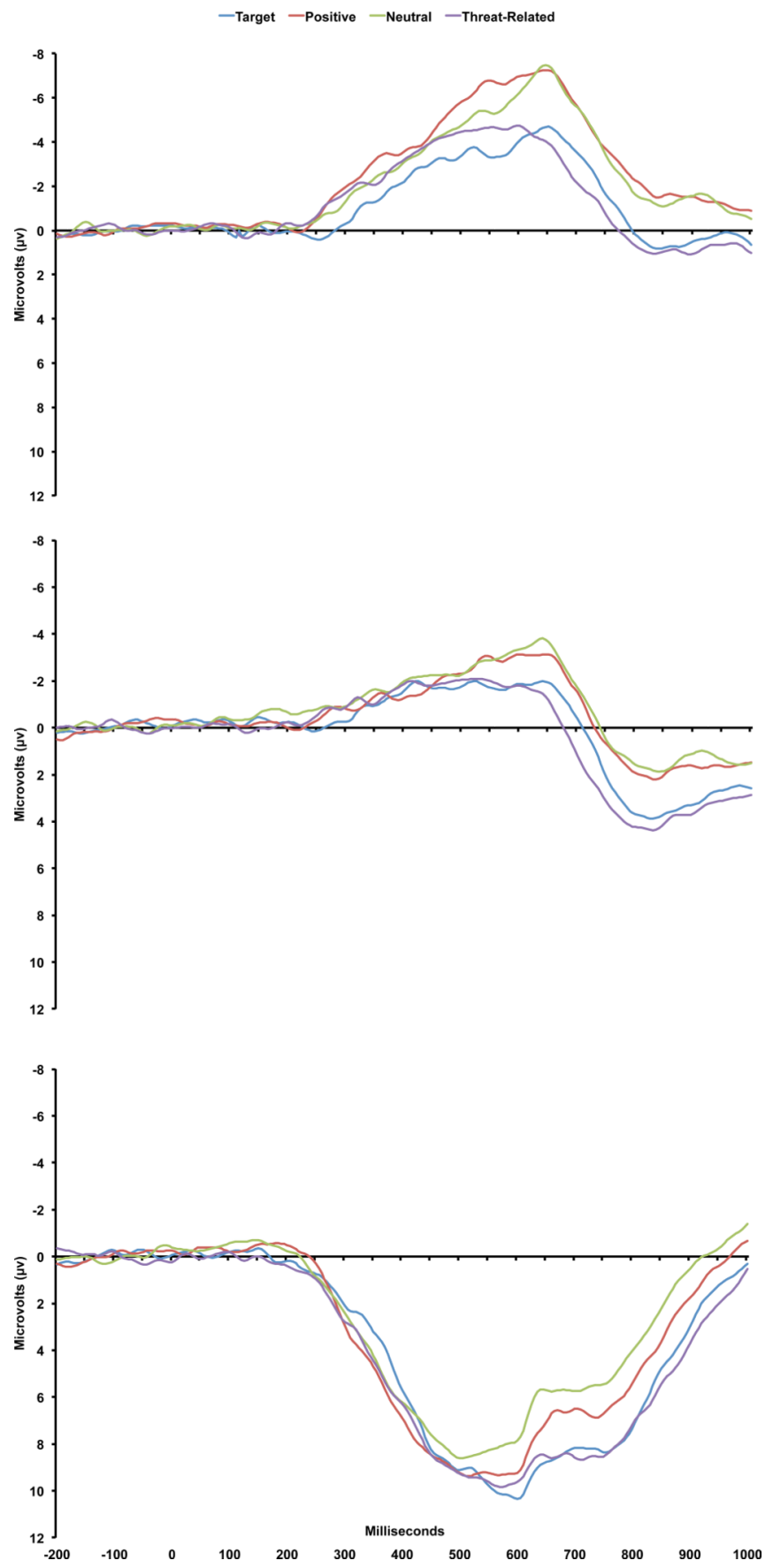

Figure 1.

Raw ERP waveforms at Fz, Cz, and Pz elicited by target, positive, neutral, and threat-related pictures presented in the context of a modified oddball task. 


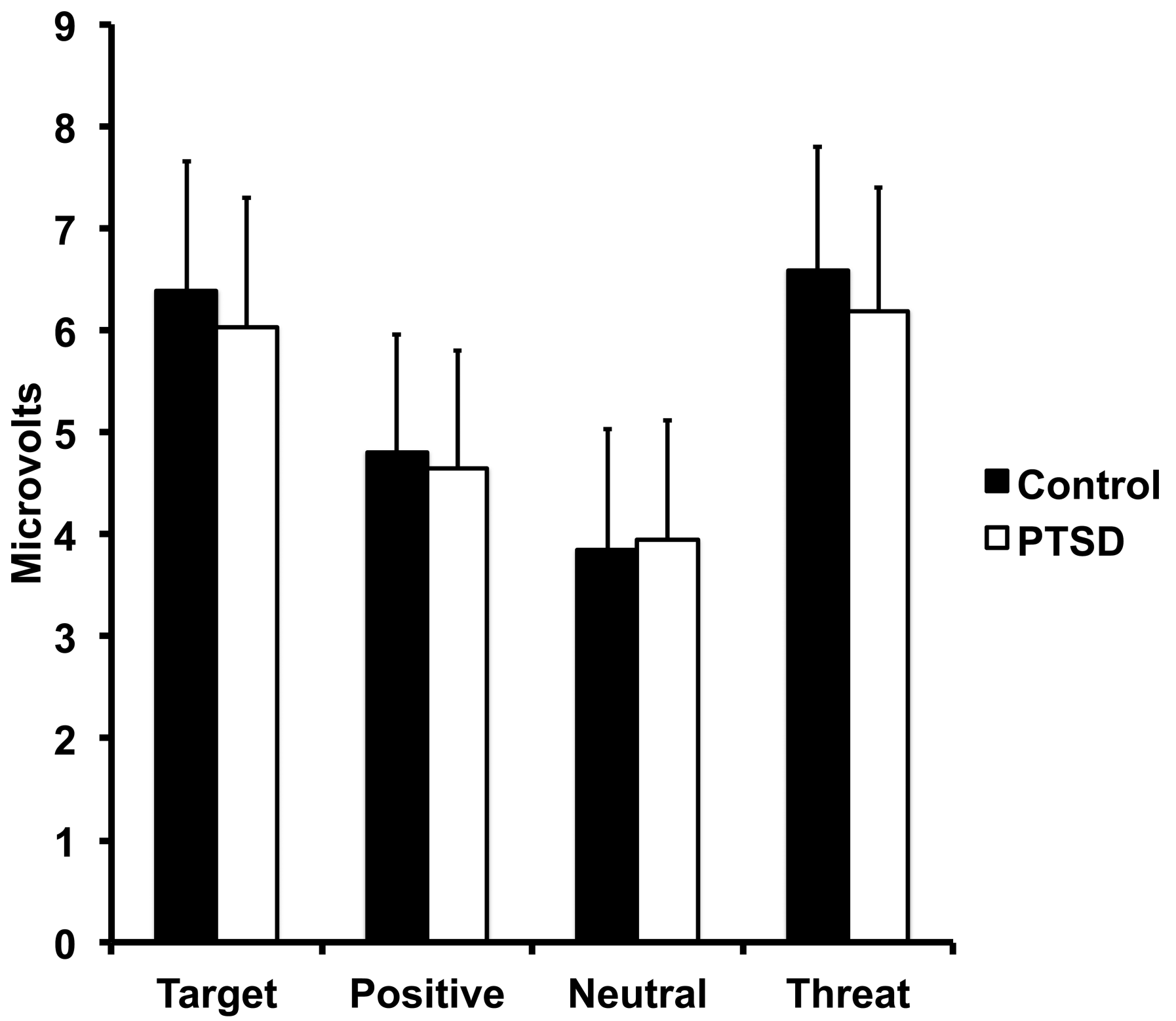

Figure 2.

LPP Responses at Pz elicited by pictures in the oddball task. Time one and time two data are averaged. 
Control Group

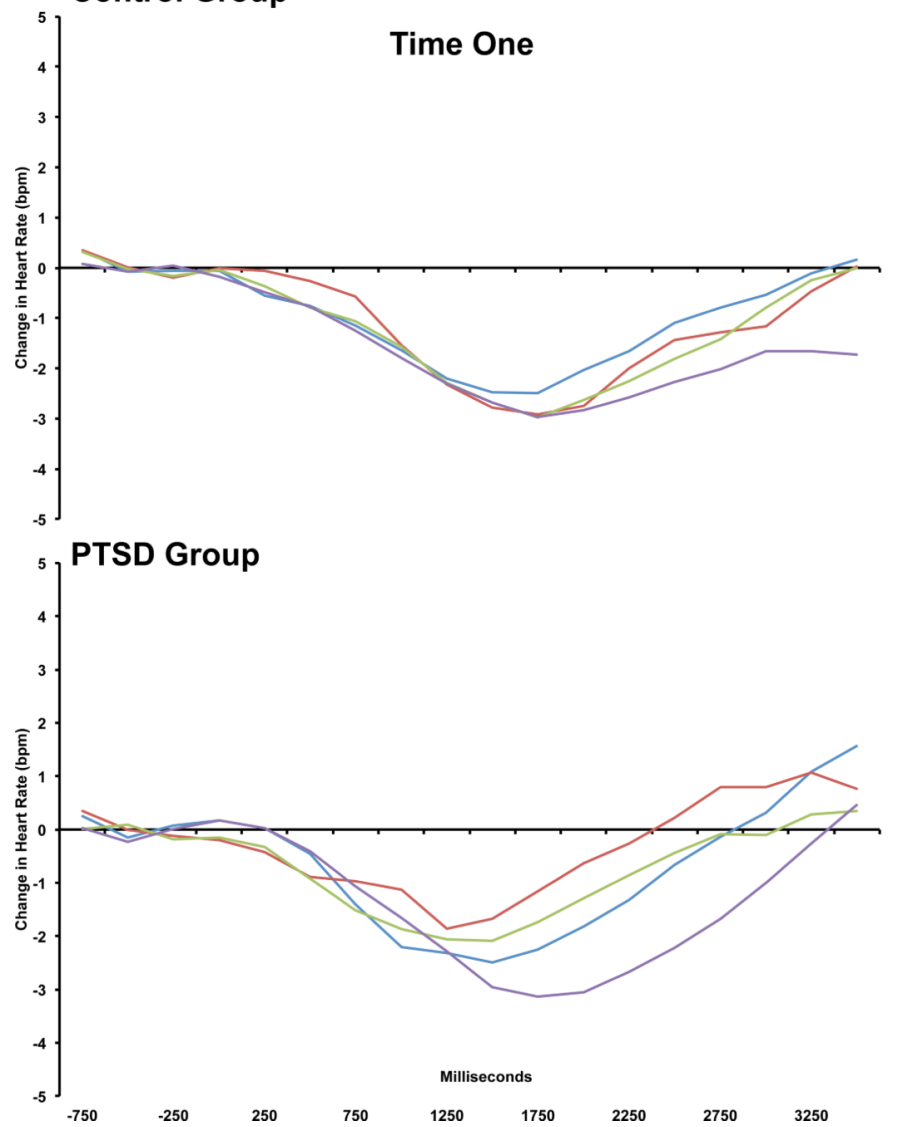

-Target - Positive - Neutral -Threat

Time Two
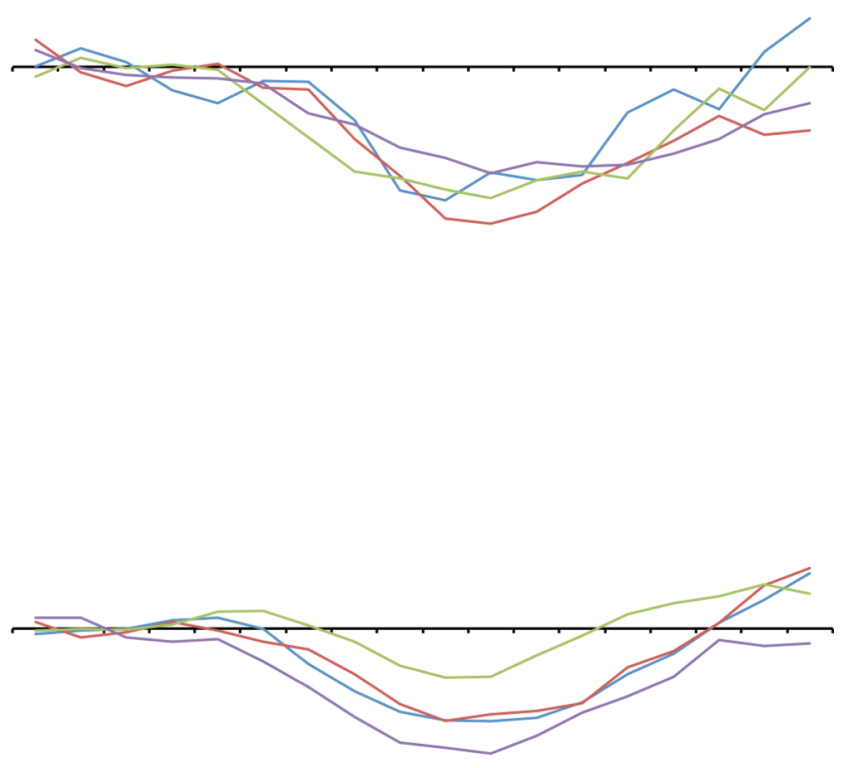

$\begin{array}{rrr}-750 & -250 \quad 250 \quad 750\end{array}$
Milliseconds

Figure 3.

Raw HRR waveforms elicited by target, positive, neutral, and threat-related pictures presented in the context of a modified oddball task. 


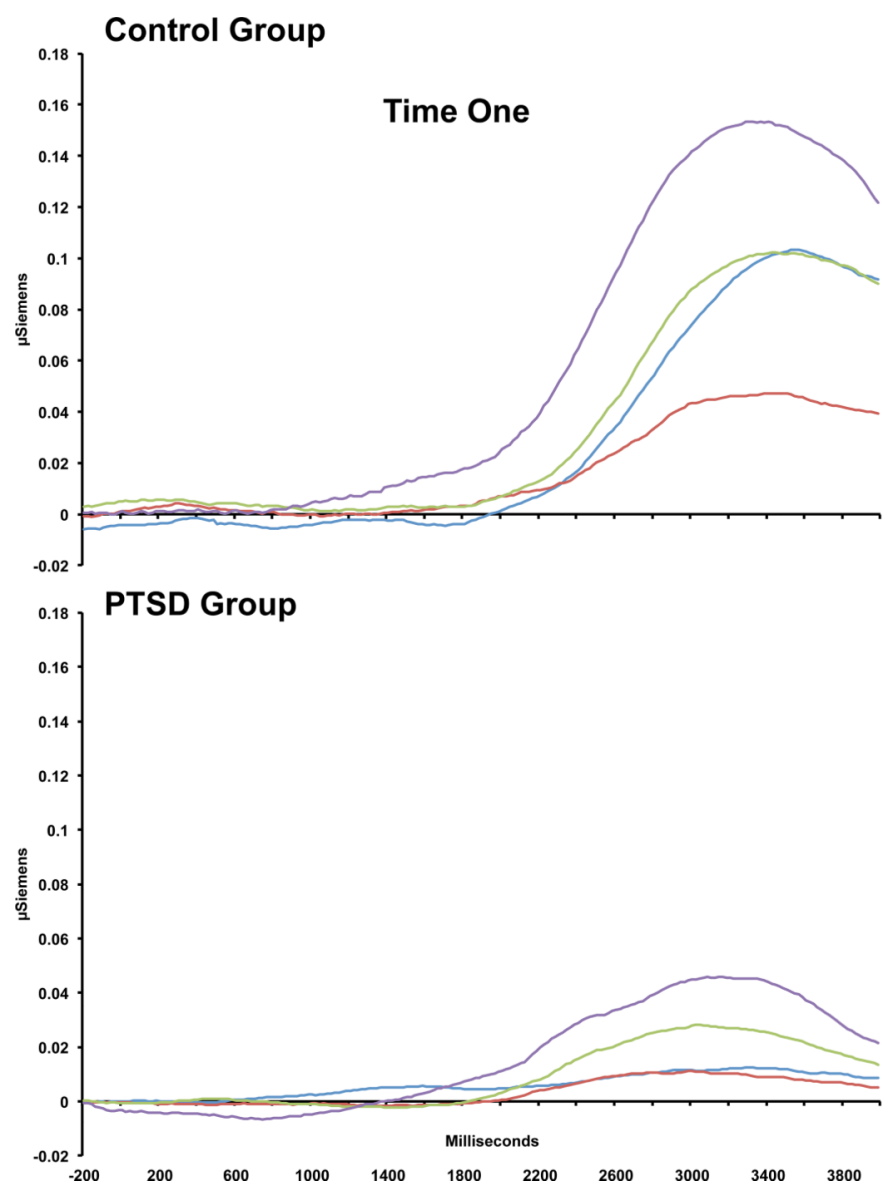

-Target -Positive - Neutral -Threat

Time Two

Figure 4.

Raw SCR waveforms elicited by target, positive, neutral, and threat-related pictures presented in the context of a modified oddball task. 

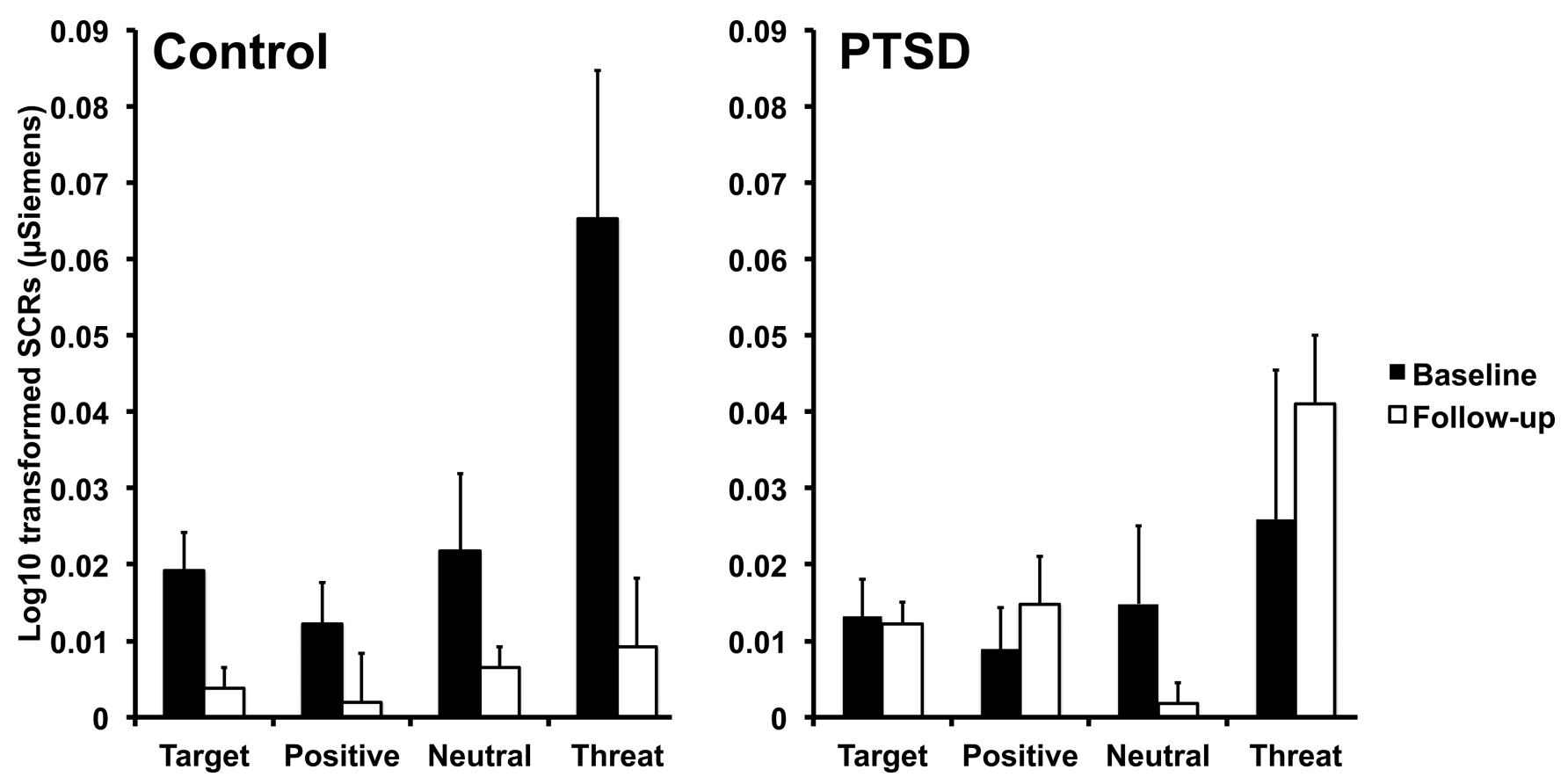

Figure 5.

SCRs to pictures in the oddball task at time one and time two. SCR data is $\log 10$ transformed. 